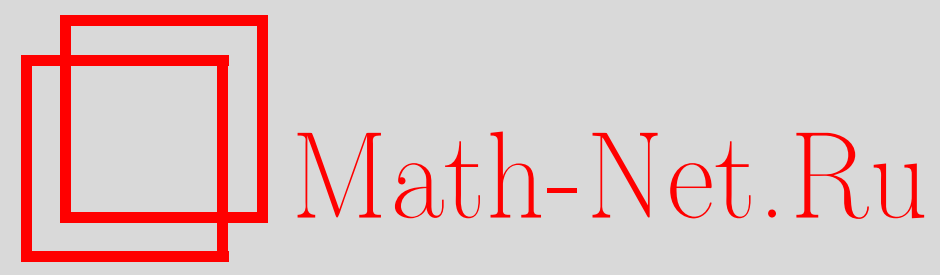

E. Clement, Bias correction for the estimation of discretized diffusion processes from an approximated likelihood function, Теория вероятн. и ее примен., 1997, том 42, выпуск 2, 364370

DOI: https://doi.org/10.4213/tvp1810

Использование Общероссийского математического портала Math-Net.Ru подразумевает, что вы прочитали и согласны с пользовательским соглашением http://www . mathnet.ru/rus/agreement

Параметры загрузки:

IP : 54.162 .127 .20

26 апреля 2023 г., 14:07:54

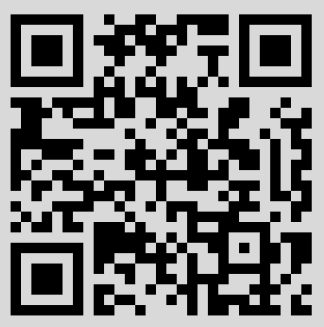


7. Drewnowski $L$. Boundedness of vector measures with values in the spaces $L_{0}$ of Bochner measurable function. - Proc. Amer. Math. Soc., 1984, v. 91, № 4, p. 581588.

8. Drewnowski L. Topological rings of sets, continuous set functions, integration. I. Bull. Acad. Polon. Sci. Ser. Sci. Math. Astr. Phys., 1972, v. 20, Na 4, p. 269-276.

Поступила в редакцию 23.VI.1995

(c) $1997 \mathbf{r}$.

\author{
CLEMENT E.*
}

\title{
BIAS CORRECTION FOR THE ESTIMATION OF DISCRETIZED DIFFUSION PROCESSES FROM AN APPROXIMATED LIKELIHOOD FUNCTION
}

\begin{abstract}
Проводится оценивание параметров дискретизированного диффузионного процесса, основанное на аппроксимации функции правдоподобия. Предполагается, что шаг между двумя последовательными наблюдениями пропесса фиксирован. Для корректировки смешения оценок предлагается использовать имитации проuecca.
\end{abstract}

Ключевые слова и Фразы: пиффузнонный процесс, схема дискретизации, схема Эйлера, асимптотические свойства оценок.

1. Notation. We consider the univariate diffusion process $\left(X_{t}\right)$, the solution of the stochastic differential equation:

$$
d X_{t}=b\left(\theta, X_{t}\right) d t+a\left(\theta, X_{t}\right) d W_{t}, \quad X_{0}=x_{0},
$$

where $W_{t}$ is a standard brownian motion, $\theta$ is a parameter belonging to a compact subset $\Theta$ of $\mathbf{R}^{p}, a$ and $b$ are two known functions. We are interested in estimating $\theta$ from discrete observations of the process $\left(X_{i_{i}}\right)_{0 \leqslant i \leqslant n}$, with a step between two consecutive observations $\Delta=t_{i+1}-t_{i}$ fixed. Since the transition probability of the homogeneous Markov process $\left(X_{t_{i}}\right)$ has an untractable form (see [1]), we will not estimate $\theta$ by a maximum likelihood method but we will maximize an approximation of the likelihood function. Since we assume that the observation step is fixed, such estimators are biased (see [4]) and we propose here to correct the bias of these estimators from simulations of the process following the method proposed by Gouriéroux-Monfort-Renault [2].

We denote by $\theta_{0}$ the true parameter value. We assume that $a$ and $b$ are smooth enough such that equation (1) admits a unique weak solution for the true value $\theta_{0}$ of parameter (see [5]) and we denote by $P_{x_{0}}^{\theta_{0}}$ the distribution of this solution on the space of continuous functions from $\mathbf{R}^{+}$into $\mathbf{R}$ equipped with the canonical filtration. $P^{W}$ stands for the brownian measure. We denote by $p_{\Delta}^{\theta}(x, y)$ and $\pi^{\theta}(x)$ respectively the densities of the transition probability at step $\Delta$ and the invariant measure of the process solution of (1) for any parameter value $\theta$.

From the observations $\left(X_{t_{i}}\right)_{0 \leqslant i \leqslant n}$ of the process, we construct a first step estimator:

$$
F_{n}=\arg \min _{\beta \in \Theta} L_{n}(\beta),
$$

\footnotetext{
*E.N.S.A.E. 3, Avenue Pierre Larousse, 92245 Malakoff Cedex, France.
} 
with

$$
L_{n}(\beta)=\frac{1}{n} \sum_{i=0}^{n-1}\left(\log a\left(\beta, X_{t_{i}}\right)+\frac{\left(X_{t_{i+1}}-X_{t_{i}}-b\left(\beta, X_{i_{i}}\right) \Delta\right)^{2}}{2 a\left(\beta, X_{t_{i}}\right)^{2}}\right)
$$

This first step estimator $F_{n}$ is obtained by minimizing the opposite of the likelihood function of the Euler approximation at step $\Delta$ of the process solution of (1), defined by

$$
X_{t_{i+1}}-X_{t_{i}}=b\left(\theta, X_{t_{i}}\right) \Delta+a\left(\theta, X_{t_{i}}\right)\left(W_{t_{i+1}}-W_{t_{i}}\right) \text {. }
$$

Since this Euler approximation converges in distribution to the process solution of $(1)$ as $\Delta$ tends to zero, the contrast $L_{n}(\beta)$ can be viewed as an approximation of the likelihood function.

We note

$$
L(\theta, \beta)=\int \log a(\beta, x) \pi^{\theta}(x) d x+\iint \frac{(y-x-b(\beta, x) \Delta)^{2}}{2 a(\beta, x)^{2}} p_{\Delta}^{\theta}(x, y) \pi^{\theta}(x) d y d x .
$$

We assume that for all $\theta$ in $\Theta$, the function $L(\theta,$.$) admits F(\theta)$ as its unique minimum:

$$
F(\theta)=\arg \min _{\beta \in \Theta} L(\theta, \beta) .
$$

Finally, we denote by $l(\beta, x, y)$ the function

$$
l(\beta, x, y)=\log a(\beta, x)+\frac{(y-x-b(\beta, x) \Delta)^{2}}{2 a(\beta, x)^{2}} .
$$

We make the following hypotheses:

$\mathbf{H}_{0}: \Theta$ is compact and $\theta_{0}$ is an interior point of $\Theta$.

$\mathbf{H}_{1}: x \mapsto b(\theta, x)$ and $x \mapsto a(\theta, x)$ are $\mathcal{C}^{\infty}$ for each $\theta$ in $\Theta$ and all their derivatives are bounded; $a$ is bounded; $a(\theta, x)>a>0$ for each $(\theta, x)$ in $\Theta \times \mathbf{R}$; there exists a compact subset $K$ and a strictly positive real $\gamma$ such that $x b(\theta, x) \leqslant-\gamma x^{2}$, for all $x$ not in $K$.

$\mathbf{H}_{2}: \theta \mapsto b(\theta, x)$ and $\theta \mapsto a(\theta, x)$ are $\mathcal{C}^{3}$ for each $x$ in $\mathbf{R}$ and their derivatives have at most polynomial growth with respect to $x$ uniformly in $\theta ;(\theta, \beta) \longmapsto(\partial L / \partial \beta)(\theta, \beta)$ is $\mathcal{C}^{2} ; F$ is $\mathcal{C}^{2}$; the matrices $D F\left(\theta_{0}\right)$ and $\left(\partial^{2} L / \partial \beta^{2}\right)\left(\theta_{0}, F\left(\theta_{0}\right)\right)$ are positive definite.

$\mathbf{H}_{3}: F(\theta)=F\left(\theta_{0}\right)$ if and only if $\theta=\theta_{0}$.

$\mathbf{H}_{\mathbf{4}}: F$ is a $\mathcal{C}^{1}$-diffeomorphism.

We have the following result for the first step estimator defined by (2).

Proposition 1. Under $\mathrm{H}_{0}, \mathrm{H}_{1}$ and $\mathrm{H}_{2}$, the first step estimator $F_{n}$ converges almost surely under $P_{x_{0}}^{\theta_{0}}$ to $F\left(\theta_{0}\right)$.

$\mathrm{P} \mathrm{r} \circ \circ \mathrm{f}$. From the ergodicity assumption $\mathrm{H}_{1}$, we obtain the almost sure convergence under $P_{x_{0}}^{\theta_{0}}$ of $L_{n}(\beta)$ to $L\left(\theta_{0}, \beta\right)$ for each $\beta$ in $\Theta$. The Lipschitz conditions on the coefficients $a$ and $b$ of the process (assumption $\mathbf{H}_{2}$ ) imply the almost sure convergence of $\sup _{\beta}\left|\left(\partial L_{n} / \partial \beta\right)(\beta)\right|$, and this implies in particular that $\sup _{\beta}\left|\left(\partial L_{n} / \partial \beta\right)(\beta)\right|$ is bounded independently of $n$, almost surely. We deduce then the almost sure equicontinuity of the family of functions $L_{n}(\beta)$ and as a consequence of Ascoli's theorem the almost sure uniform convergence of $L_{n}(\beta)$ for $\beta$ in $\Theta$. Since $F\left(\theta_{0}\right)$ is the unique minimum point of $L\left(\theta_{0},.\right)$ we obtain the almost sure convergence of $F_{n}$ to $F\left(\theta_{0}\right)$.

This first step estimator is then biased and we will see in the next sections how to correct this estimator.

$\mathrm{E} \times \mathrm{a} \mathrm{m} \mathrm{pl}$ e. Consider the Ornstein-Uhlenbeck process, the solution of

$$
d X_{t}=-\mu X_{t} d t+\sigma d W_{t}, \quad X_{0}=0,
$$

where $\theta=\left(\mu, \sigma^{2}\right)^{t}$ is the unknown parameter value. We suppose that $\mu$ and $\sigma$ are strictly positive. The process $X_{t}$ is then ergodic, $\pi^{\theta}$ is the density of the normal distribution 
$\mathcal{N}\left(0, \sigma^{2} /(2 \mu)\right)$ and $p_{\Delta}^{\theta}(x, \cdot)$ is the density of the normal distribution $\mathcal{N}\left(\exp (-\mu \Delta) x, \sigma^{2}(1-\right.$ $\exp (-2 \mu \Delta)) /(2 \mu))$. For this process, we can estimate $\theta$ by a maximum likelihood method but it is no more the case for more complicated processes. Suppose then that we estimate $\theta$ by minimizing in $\beta=\left(\beta_{1}, \beta_{2}\right)^{t}$ the following contrast:

$$
\frac{1}{n} \sum_{i=0}^{n-1}\left[\frac{1}{2} \log \left(2 \pi \beta_{2} \Delta\right)+\frac{1}{2 \beta_{2} \Delta}\left(X_{t_{i+1}}-\left(1-\beta_{1} \Delta\right) X_{i_{i}}\right)^{2}\right]
$$

which is the opposite of the likelihood function of the Euler discretization scheme of the process $\left(X_{t_{i}}\right)$ solution of (3):

$$
X_{t_{i+1}}-X_{t_{i}}=-\mu X_{t_{i}} \Delta+\sigma\left(W_{t_{i}+1}-W_{t_{i}}\right) .
$$

We obtain a first step estimator $F_{n}=\left(\tilde{\mu}_{n}, \tilde{\sigma}_{n}^{2}\right)^{t}$ which is biased if we assume $\Delta$ fixed. We have

$$
\lim _{n \rightarrow+\infty} F_{n}=F(\theta)=\left(\begin{array}{c}
\frac{1-\exp (-\mu \Delta)}{\Delta} \\
\frac{\sigma^{2}}{2 \mu \Delta}(1-\exp (-2 \mu \Delta))
\end{array}\right) .
$$

2. An auxiliary estimator. We will first consider that the function $F(\theta)$ is known (this is the case for the Ornstein-Uhlenbeck process) and define the estimator

$$
\hat{\theta}_{n}=\arg \min _{\theta \in \Theta}\left\|F_{n}-F(\theta)\right\|
$$

where $\|\cdot\|$ denotes the Euclidean norm. This estimator has just a theoretical interest since the function $F(\theta)$ depends on the transition probability of the process and is generally unknown. We have the result:

Proposition 2. Under $\mathrm{H}_{0}-\mathrm{H}_{3}$, we have

i) $\lim _{n \rightarrow+\infty} \hat{\theta}_{n}=\theta_{0}$ a.s. under $P_{x_{0}}^{\theta_{0}}$,

ii) $\sqrt{n}\left(\hat{\theta}_{n}-\theta_{0}\right) \Longrightarrow \mathcal{N}\left(0, D F\left(\theta_{0}\right)^{-1} \widetilde{\Sigma} D F^{t}\left(\theta_{0}\right)^{-1}\right), n \rightarrow+\infty$, with

$$
\begin{aligned}
\widetilde{\Sigma}= & \frac{\partial^{2} L}{\partial \beta^{2}}\left(\theta_{0}, F\left(\theta_{0}\right)\right)^{-1} \Sigma^{0}\left(\frac{\partial l}{\partial \beta}\right) \frac{\partial^{2} L}{\partial \beta^{2}}\left(\theta_{0}, F\left(\theta_{0}\right)\right)^{-1} \\
\Sigma^{0}(f)= & \iint\left(f(x, y)-\mathbf{E}_{p_{\Delta}^{\theta_{0}} \theta_{0}} f\right)\left(f(x, y)-\mathbf{E}_{p_{\Delta}^{\theta_{0}} \pi_{0}^{\theta_{0}}} f\right)^{t} p_{\Delta}^{\theta_{0}}(x, y) \pi^{\theta_{0}}(x) d y d x \\
& +2 \int\left(\int\left(f(x, y)-\mathbf{E}_{p_{\Delta}^{\theta_{0}} \pi_{0}} f\right) p_{\Delta}^{\theta_{0}}(x, y) d y\right) G^{t}(x) \pi^{\theta_{0}}(x) d x \\
G(x)= & \sum_{k \geqslant 1} \int\left(f(x, y)-\mathbf{E}_{p_{\Delta}^{\theta_{0}} \pi^{\theta_{0}}} f\right) p_{k \Delta}^{\theta_{0}}(x, y) d y .
\end{aligned}
$$

P r o o f. i) From Proposition 1, we have

$$
\lim _{n \rightarrow+\infty} F_{n}=F\left(\theta_{0}\right) \text { a.s. }
$$

We can deduce that

$$
\lim _{n \rightarrow+\infty}\left\|F_{n}-F(\theta)\right\|=\left\|F\left(\theta_{0}\right)-F(\theta)\right\| \quad P_{x_{0}}^{\theta_{0}-\text { a.s. }}
$$

Moreover the family of functions $\left(\left\|F_{n}-F(\theta)\right\|\right)$ is equicontinuous since from $\mathrm{H}_{0}$ and $\mathbf{H}_{2}$ it follows

$$
\left|\left\|F_{n}-F(\theta)\right\|-\left\|F_{n}-F\left(\theta^{\prime}\right)\right\|\right| \leqslant \sup _{\theta \in \Theta}\|D F(\theta)\|\left\|\theta-\theta^{\prime}\right\|
$$


This implies the almost sure convergence under $P_{x_{0}}^{\theta_{0}}$ of $\left\|F_{n}-F(\theta)\right\|$ as $n$ tends to infinity. From $\mathbf{H}_{3}$, the limit function $\left\|F\left(\theta_{0}\right)-F(\theta)\right\|$ has its minimum at the unique point $\theta_{0}$, this implies the almost sure convergence of $\hat{\theta}_{n}$.

ii) We denote by $\varphi_{n}(\theta)$ the function $\left(F_{n}-F(\theta)\right)^{t}\left(F_{n}-F(\theta)\right)$. Since $\varphi_{n}(\theta)$ is $\mathcal{C}^{2}$ $\left(\mathrm{H}_{2}\right)$ and $\theta_{0}$ belongs to the interior of $\Theta\left(\mathrm{H}_{0}\right)$, we can expand it around $\theta_{0}$, on the set $\left\{\hat{\theta}_{n} \in \dot{\Theta}\right\}$, whose probability tends to 1 since from i) $\hat{\theta}_{n}$ tends to $\theta_{0}$ almost surely, we have then

$$
D \varphi_{n}\left(\hat{\theta}_{n}\right)=D \varphi_{n}\left(\theta_{0}\right)+\int_{0}^{1} D^{2} \varphi_{n}\left(\theta_{0}+u\left(\hat{\theta}_{n}-\theta_{0}\right)\right) d u \cdot\left(\hat{\theta}_{n}-\theta_{0}\right)
$$

with $D \varphi_{n}\left(\hat{\theta}_{n}\right)=0$.

This expansion can be expressed in function of $F$ and its differentials of order one and two, we obtain

$$
\begin{gathered}
D F\left(\theta_{0}\right)^{t} \cdot\left(F_{n}-F\left(\theta_{0}\right)\right)=\int_{0}^{1} D F^{t}\left(\theta_{0}+u\left(\hat{\theta}_{n}-\theta_{0}\right)\right) D F\left(\theta_{0}+u\left(\hat{\theta}_{n}-\theta_{0}\right)\right) \cdot\left(\hat{\theta}_{n}-\theta_{0}\right) d u \\
\quad+\int_{0}^{1} D^{2} F\left(\theta_{0}+u\left(\hat{\theta}_{n}-\theta_{0}\right)\right) \cdot\left(F_{n}-F\left(\theta_{0}+u\left(\hat{\theta}_{n}-\theta_{0}\right)\right), \hat{\theta}_{n}-\theta_{0}\right) d u
\end{gathered}
$$

Since $F$ is $\mathcal{C}^{2}$ and $\Theta$ is compact and since from i) $\hat{\theta}_{n}$ converges to $\theta_{0}$ almost surely we obtain the convergence by Lebesgue's theorem:

$$
\begin{aligned}
& \lim _{n \rightarrow+\infty} \int_{0}^{1} D F^{t}\left(\theta_{0}+u\left(\hat{\theta}_{n}-\theta_{0}\right)\right) D F\left(\theta_{0}+u\left(\hat{\theta}_{n}-\theta_{0}\right)\right) d u=D F^{t}\left(\theta_{0}\right) D F\left(\theta_{0}\right), \\
& \lim _{n \rightarrow+\infty} \int_{0}^{1} D^{2} F\left(\theta_{0}+u\left(\hat{\theta}_{n}-\theta_{0}\right)\right) \cdot\left(F_{n}-F\left(\theta_{0}+u\left(\hat{\theta}_{n}-\theta_{0}\right)\right), 1\right) d u=0 .
\end{aligned}
$$

It suffices to prove the asymptotic normality of $\sqrt{n}\left(F_{n}-F\left(\theta_{0}\right)\right)$ with

$$
F_{n}=\arg \min _{\beta} L_{n}(\beta) \text {. }
$$

An expansion of the derivative with respect to $\beta$ of the function $L_{n}(\beta)$ around $F\left(\theta_{0}\right)$ gives

$$
\int_{0}^{1} \frac{\partial^{2} L_{n}}{\partial \beta^{2}}\left(F\left(\theta_{0}\right)+u\left(F_{n}-F\left(\theta_{0}\right)\right)\right) d u .\left(F_{n}-F\left(\theta_{0}\right)\right)=-\frac{\partial L_{n}}{\partial \beta}\left(F\left(\theta_{0}\right)\right) .
$$

From assumption $\mathbf{H}_{2}$, the function $(\partial l / \partial \beta)(\beta, x, y)$ belongs to $L^{2}\left(p_{\Delta}^{\theta_{0}} \pi^{\theta_{0}}\right)$. Applying the result of Dacuhna-Castelle and Florens-Zmirou [1], this function satisfies a central limit theorem and we have

$$
\sqrt{n} \frac{\partial L_{n}}{\partial \beta}\left(\theta_{0}, F\left(\theta_{0}\right)\right) \Longrightarrow \mathcal{N}\left(0, \Sigma^{0}\left(\frac{\partial l}{\partial \beta}\right)\right), \quad n \rightarrow+\infty
$$

where $\Sigma^{0}(\partial l / \partial \beta)$ is defined in Proposition 2. Moreover we have the almost sure convergence of $\sup _{\beta}\left|\left(\partial^{2} L_{n} / \partial \beta^{2}\right)(\beta)\right|$ from the Lipschitz conditions on derivatives of order one and two of coefficients $a$ and $b$. We deduce then

$$
\lim _{n \rightarrow+\infty} \int_{0}^{1} \frac{\partial^{2} L_{n}}{\partial \beta^{2}}\left(F\left(\theta_{0}\right)+u\left(F_{n}-F\left(\theta_{0}\right)\right)\right) d u=\frac{\partial^{2} L}{\partial \beta^{2}}\left(F\left(\theta_{0}\right)\right)
$$

Finally we obtain

$$
\sqrt{n}\left(F_{n}-F\left(\theta_{0}\right)\right) \Longrightarrow \mathcal{N}(0, \tilde{\Sigma}), \quad n \rightarrow+\infty
$$


where $\tilde{\Sigma}$ is defined in Proposition 2 and Proposition 2 is proved.

3. Sirnulated estimator. Since $F(\theta)$ is unknown we will approximate it from simulated observations. For a value of parameter $\theta$, we will consider the process $\left(X_{t, h}^{\theta, s}\right)$ solution of the Euler dicretization scheme with a discretization step equal to $\Delta / h$ :

$$
X_{t+\Delta / h, h}^{\theta, s}-X_{t, h}^{\theta, s}=b\left(\theta, X_{t, h}^{\theta, \theta}\right) \frac{\Delta}{h}+a\left(\theta, X_{t}^{\theta, \Delta}\right)\left(W_{t+\Delta / h}^{s}-W_{t}^{s}\right), \quad X_{0, h}^{\theta, s}=x,
$$

where $W^{s}$ is a brownian motion independent of $W$. We can then construct $N$ simulated observations $\left(X_{t_{i}, h}^{\theta, s}\right)_{0 \leqslant i \leqslant N}$. From these simulated observations, we define a first step simulated estimator $F_{N}^{h}(\theta)$ :

$$
F_{N}^{h}(\theta)=\arg \min _{\beta \in \Theta} L_{N}^{h}(\theta, \beta)
$$

where

$$
L_{N}^{h}(\theta, \beta)=\frac{1}{N} \sum_{i=0}^{N-1}\left(\log a\left(\beta, X_{i_{i}, h}^{\theta, s}\right)+\frac{\left(X_{t_{i+1}, h}^{\theta, s}-X_{i_{i, h}}^{\theta, s}-b\left(\beta, X_{t_{i, h}}^{\theta, s}\right) \Delta\right)^{2}}{2 a\left(\beta, X_{t_{i, h}}^{\theta, s}\right)^{2}}\right) .
$$

In practice, such trajectories of the process can be simulated for a finite number of parameter values. We consider then a regular grid $\Theta_{m}$ of $\Theta$ made of $m^{p}$ points and we define the simulated estimator as follows:

$$
\hat{\theta}_{n, N}^{m, h}=\arg \min _{\theta \in \Theta_{m}}\left\|F_{n}-F_{N}^{h}(\theta)\right\|
$$

We will then determine $m, N$ and $h$ as functions of $n$ such that the simulated estimator $\bar{\theta}_{n, N}^{m, h}$ inherits the same asymptotic properties as the auxiliary estimator $\hat{\theta}_{n}$.

Proposition 3. Let $m(n)$ be a sequence such that

$$
\lim _{n \rightarrow+\infty} \frac{\sqrt{n}}{m(n)}=0
$$

then, under $\mathrm{H}_{0}, \mathrm{H}_{1}, \mathrm{H}_{2}$ and $\mathrm{H}_{4}$ there exist sequences $h(n)$ and $N(n)$ such that

$$
\sqrt{n}\left(\hat{\theta}_{n, N(n)}^{m(n), R(n)}-\hat{\theta}_{n}\right) \longrightarrow 0 \text { in probability under } P_{x_{0}}^{\theta_{0}} \otimes P^{W} \otimes \delta_{x}, \quad n \rightarrow+\infty
$$

From Propositions 3 and 2, we deduce immediately the corollary.

Corollary. Under hypotheses of Proposition 3, there exist $N(n)$ and $h(n)$ such that

$$
\begin{aligned}
& \lim _{n \rightarrow+\infty} \hat{\theta}_{n, N(n)}^{m(n), h(n)}=\theta_{0} \quad \text { in probability under } \quad P_{x_{0}}^{\theta_{0}} \otimes P^{W} \otimes \delta_{x}, \\
& \sqrt{n}\left(\hat{\theta}_{n, N(n)}^{m(n), h(n)}-\theta_{0}\right) \Longrightarrow \mathcal{N}\left(0, D F\left(\theta_{0}\right)^{-1} \widetilde{\Sigma} D F^{t}\left(\theta_{0}\right)^{-1}\right), \quad n \rightarrow+\infty
\end{aligned}
$$

where $\tilde{\Sigma}$ is defined in Proposition 2.

Proof of Proposition 3. The proof of Proposition 3 is based on the following lemma.

Lemma. If $\left\|F_{N(n)}^{h(n)}(\theta)-F(\theta)\right\| \leqslant 1 / m(n)$ for every $\theta \in \Theta_{m}$ then, under $\mathbf{H}_{0}-\mathbf{H}_{4}$, we have almost surely for $n$ large enough

$$
\left\|\hat{\theta}_{n, N(n)}^{m(n), h(n)}-\hat{\theta}_{n}\right\| \leqslant \frac{C}{m(n)}
$$

where $C$ is a constant independent of $n$. 
$\mathrm{P}$ r $\circ$ o f. From $\mathrm{H}_{4}$, we obtain by a Taylor expansion of $F^{-1}$ the following inequality:

$$
\left\|\hat{\theta}_{n, N(n)}^{m(n)}-\hat{\theta}_{n}\right\| \leqslant C\left\|F\left(\hat{\theta}_{n, N(n)}^{m(n)}\right)-F\left(\hat{\theta}_{n}\right)\right\|,
$$

where $C$ is a constant. Since $F_{n}$ converges almost surely to $F\left(\theta_{0}\right)$ (Proposition 1), there exists $n_{0}$ (with $n_{0}<\infty$ almost surely) such that $\left(\forall n \geqslant n_{0}\right) F_{n}$ is in $F(\Theta)$, from $\mathrm{H}_{4}$ it yields $F\left(\hat{\theta}_{n}\right)=F_{n}$, and then

$$
\left\|\hat{\theta}_{n, N(n)}^{m(n)}-\hat{\theta}_{n}\right\| \leqslant C \| F_{n}-F\left(\hat{\theta}_{n, N(n)}^{m(n)} \| \leqslant C\left(\frac{1}{m(n)}+\| F_{n}-F_{N(n)}\left(\hat{\theta}_{n, N(n)}^{m(n)} \|\right) .\right.\right.
$$

Now since $\Theta_{m}$ is a regular grid of $\Theta$ made of $m^{p}$ points, we can choose $\theta_{n, m}$ in $\Theta_{m}$ such that

$$
\left\|\hat{\theta}_{n}-\theta_{n, m}\right\| \leqslant \frac{C}{m(n)}
$$

but by definition of $\hat{\theta}_{n, N(n)}^{m(n)}$ and since $\theta_{n, m}$ belongs to $\Theta_{m}$, we have

$$
\left\|F_{n}-F_{N(n)}\left(\hat{\theta}_{n, N(n)}^{m(n)}\right)\right\| \leqslant\left\|F_{n}-F_{N(n)}\left(\theta_{n, m}\right)\right\|
$$

so we obtain

$$
\begin{aligned}
\left\|F_{n}-F_{N(n)}\left(\hat{\theta}_{n, N(n)}^{m(n)}\right)\right\| & \leqslant\left\|F_{n}-F_{N(n)}\left(\theta_{n, m}\right)\right\| \\
& \leqslant\left\|F\left(\hat{\theta}_{n}\right)-F\left(\theta_{n, m}\right)\right\|+\left\|F\left(\theta_{n, m}\right)-F_{N(n)}\left(\theta_{n, m}\right)\right\| \leqslant \frac{C}{m(n)},
\end{aligned}
$$

and then

$$
\left\|\dot{\theta}_{n, N(n)}^{m(n)}-\dot{\theta}_{n}\right\| \leqslant \frac{C}{m(n)}
$$

this is the result of the lemma.

From the lemma, to prove Proposition 3 it suffices to verify that

$$
\lim _{n \rightarrow+\infty} \mathbf{P}\left\{\left(\forall \theta \in \Theta_{m(n)}\right)\left\|F_{N(n)}^{h(n)}(\theta)-F(\theta)\right\| \leqslant \frac{1}{m(n)}\right\}=1
$$

or equivalently

$$
\lim _{n \rightarrow+\infty} \mathbf{P}\left\{\left(\exists \theta \in \Theta_{m(n)}\right) \text { such that }\left\|F_{N(n)}^{h(n)}(\theta)-F(\theta)\right\|>\frac{1}{m(n)}\right\}=0 .
$$

We note $F^{h}(\theta)$ the limit as $N$ tends to infinity of $F_{N}^{h}(\theta)$ (for the existence of this limit see [6]). We have

$$
\begin{aligned}
& \mathbf{P}\left\{\left(\exists \theta \in \Theta_{m}\right)\left\|F_{N(n)}^{h(n)}(\theta)-F(\theta)\right\|>\frac{1}{m(n)}\right\} \\
& \leqslant m(n)^{p} \sup _{\theta \in \Theta_{m}} \mathbf{P}\left\{\left\|F_{N(n)}^{h(n)}(\theta)-F^{h(n)}(\theta)\right\|>\frac{1}{2 m(n)}\right\} \\
& +m(n)^{p} \sup _{\Theta_{m}} \mathbf{P}\left\{\left\|F^{h(n)}(\theta)-F(\theta)\right\|>\frac{1}{2 m(n)}\right\}
\end{aligned}
$$

We note $L^{h}(\theta, \beta)$ the almost sure limit of $L_{N}^{h}(\theta, \beta)$. The expression of $L^{h}(\theta, \beta)$ is then

$$
L^{h}(\theta, \beta)=\int \log a(\beta, x) \pi^{\theta, h}(x) d x+\iint \frac{(y-x-b(\beta, x) \Delta)^{2}}{2 a(\beta, x)^{2}} p_{\Delta}^{\theta, h}(x, y) \pi^{\theta, h}(x) d y d x,
$$


where $p_{\Delta}^{\theta, h}(x, y)$ and $\pi^{\theta, h}(x)$ denote respectively the densities of the transition probability and invariant measure of the process solution of (7). From [6], we have the convergence of $L^{h}(\theta, \beta)$ to $L(\theta, \beta)$ for all $(\theta, \beta)$ and this convergence is uniform in $\beta$. We deduce then the convergence of $F^{h}(\theta)$ to $F(\theta)$ for all $\theta$. This implies the existence of a sequence $h(n)$ such that

$$
\mathbf{P}\left\{\left\|F^{h(n)}(\theta)-F(\theta)\right\|>\frac{1}{2 m(n)}\right\}=0 .
$$

Since $F_{N}^{h}(\theta)$ converges almost surely to $F^{h}(\theta)$ for all $\theta$, we can choose $N(n)$ such that

$$
\mathbf{P}\left\{\left\|F_{N(n)}^{h(n)}(\theta)-F^{h(n)}(\theta)\right\|>\frac{1}{2 m(n)}\right\}<\frac{1}{m(n)^{p+1}},
$$

and we obtain the result of Proposition 3.

Acknowlegement. The author wish to thank Valentine Genon-Catalot and Jean Jacot for helpful discussions.

\section{REFERENCES}

1. Dacunha-Castelle D., Florens-Zmirou D. Estimation of the coefficient of a diffusion from discrete observations. - Stochastics, 1986, v. 19, p. 263-284.

2. Gourieroux C., Monfort A., Renault E. Indirect inference. - J. Appl. Econometrics, 1993, v, 8, p. S85-S119.

3. Липчер P. III., II иряев A. Н. Статистика случайных процессов. М.: Наука, 1974.

4. Pedersen $A$. $R$. A new approach to maximum likelihood estimation for stochastic differential equations based on discrete observations. - Scand. J. Statist., 1995, v. 22, p. 55-71.

5. Revuz D., Yor M. Continuous Martingales and Brownian Motion. Berlin: SpringerVerlag, 1991.

6. Talay $D$., Tubaro $L$. Expansion of the global error for numerical schemes solving stochastic differential equations. - Stoch. Anal. Appl., 1990, v. 8, p. 483-509. 